\title{
Large-Scale Shake Table Test Verification of Bridge Condition Assessment Methods
}

\author{
Yangbo Chen, Ph.D. ${ }^{1}$; Maria Q. Feng, M.ASCE ${ }^{2}$; and Serdar Soyoz, Ph.D. ${ }^{3}$
}

\begin{abstract}
Methods that identify structural component stiffness degradation by pre- and postevent low amplitude vibration measurements, based on a linear time-invariant (LTI) system model, are conceptually justified by examining the hysteresis loops the structural components experience in such vibrations. Two large-scale shake table experiments, one on a two-column reinforced concrete (RC) bridge bent specimen, and the other on a two-span three-bent RC bridge specimen were performed, in which specimens were subjected to earthquake ground motions with increasing amplitude and progressively damaged. In each of the damaged stages between two strong motions, low amplitude vibrations of the specimens were aroused, and the postevent component stiffness coefficients were identified by optimizing the parameters in a LTI model. The stiffness degradation identified is consistent with the experimental hysteresis, and could be quantitatively related to the capacity residual of the components.
\end{abstract}

DOI: 10.1061/(ASCE)0733-9445(2008)134:7(1235)

CE Database subject headings: Shake table tests; Verification; Bridges, concrete; Concrete, reinforced; Assessment; Vibration; Stiffness.

\section{Introduction}

Civil engineering structural components, especially those comprising reinforced concrete, when subjected to strong earthquakes or other large cyclic deformation, exhibit significant hysteresis behaviors. Hysteresis is not only a highly nonlinear phenomenon, but also a process that possesses memory. Recently, considerable efforts in the structural health monitoring research community have been endeavored to track the structural conditions (e.g., the instantaneous stiffness and damping at every instance) by identifying the hysteresis from global vibration measurements during a strong cyclic deformation (e.g., Smyth et al. 2002; Yang and Lin 2005). This has been found to be a highly challenging problem because of the nonlinearity of the system and the dependency of the current nonlinearity on previous deformation conditions. From an infrastructure maintenance point of view, however, the major concern of structural condition assessment is the postevent

${ }^{1}$ Englekirk Partners Consulting Structural Engineers Inc., Los Angeles, CA 90018; formerly, Graduate Student, Dept. of Civil and Environmental Engineering, Univ. of California, Irvine, CA 92697-2175. E-mail: yangbo.chen@englekirk.com

${ }^{2}$ Professor, Dept. of Civil and Environmental Engineering, Univ. of California, Irvine, CA92697-2175 (corresponding author). E-mail: mfeng@uci.edu

${ }^{3}$ Post-Doctoral Researcher, Dept. of Civil and Environmental Engineering, Univ. of California, Irvine, CA92697-2175; formerly, Graduate Student, Dept. of Civil and Environmental Engineering, Univ. of California, Irvine, CA 92697-2175. E-mail: ssoyoz@uci.edu

Note. Associate Editor: Ahmet Emin Aktan. Discussion open until December 1, 2008. Separate discussions must be submitted for individual papers. To extend the closing date by one month, a written request must be filed with the ASCE Managing Editor. The manuscript for this paper was submitted for review and possible publication on March 30, 2006; approved on October 8, 2007. This paper is part of the Journal of Structural Engineering, Vol. 134, No. 7, July 1, 2008. CASCE, ISSN 07339445/2008/7-1235-1245/\$25.00. status of structures, with less emphasis on the timing of damage occurrences or the progressive evolution of damages during an event. Therefore, a simpler method is favorable if it can reveal the pre- and postevent status of a structure based on vibration records.

In this paper, hysteresis loops a structure experiences in low amplitude vibration are examined following an established hysteresis model. This provides conceptual justification for the use of methods assuming a linear and time-invariant (LTI) system in analyzing the pre- and postevent low amplitude vibration data. Such assumption is also shown to be supported by experimental hysteresis curves.

A series of large scale shaking table tests have been and more will be conducted at the University of Nevada, Reno (UNR), in NSF-NEES sponsored research to explore the seismic behaviors of reinforced concrete bridges and potential design philosophy or technology improvements. Taking advantage of this opportunity, the writers collaborated with researchers in UNR and installed additional accelerometers on the bridge specimen to verify the proposed methodology of structural condition assessment for bridge bents using pre- and postevent vibration data.

The identified pre- and postevent structural conditions by a parameter optimization routine that is based on a LTI system model show good consistency with the experimental observations.

In these experiments, pre- and postevent vibrations were aroused by driving the shaking tables with low amplitude white noises or in some cases, ambient signals obtained on an instrumented bridge. On real-life structures instrumented for structural health monitoring purposes, low amplitude vibration data suitable for the proposed pre- and postevent structural assessment method are available in the forms of, e.g., ambient vibration records before and after extreme events, minor or moderate earthquake records in which no significant structural degradation is observed, or the portions of strong earthquake records that exhibit low vibration amplitude. Given the availability of such data, the writers believe that the proposed method shall be widely applicable. 


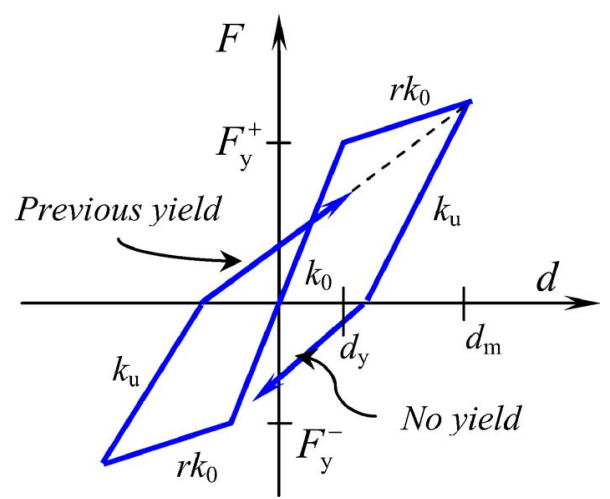

Fig. 1. Bilinear Takeda hysteresis model

\section{Hysteresis in Low-Amplitude Vibrations}

Many hysteresis models have been developed (e.g., Bouc 1967; Clough 1966; Takeda et al. 1970; Wen 1976) to characterize the force-deformation relation of a structural component in cyclic loading. Efforts have been made to present these models in a holistic manner (Sain et al. 1997; Sivaselvan and Reinhorn 2000). Among them, the Takeda model is one of the most used. It is accepted as a realistic model because it was experimentally developed and verified (Takeda et al. 1970). The detailed empirical rules in the original Takeda model have been simplified and modified aiming at a more efficient numerical implementation and a better account of phenomena such as pinching (e.g., Emori and Schnobrich 1981; Saiidi 1982; Takayanagi and Schnobrich 1979). In Park et al. (1987), it was found that the stiffness degradation can be accurately predicted by the pivot rule, according to which the load-reversal branches are targeting a pivot point on the opposite side. In terms of this rule, the bilinear Takeda model shown in Fig. 1 can be described as: (1) loading/reloading rule-if the structure has never yielded in the loading direction, the pivot point is the yielding point; otherwise, it is the largest excursion point (the maximum deformation ever reached in this direction); and (2) unloading rule - the elastic unloading slope is the same as the initial stiffness $k_{0}$, while the postyielding unloading follows a slope of $k_{u}=k_{0}\left(d_{y} / d_{m}\right)^{0.5}$, where $d_{y}$ and $d_{m}=$ yielding and largest excursion deformations, respectively.

Following this hysteresis model, the force-deformation paths under low-amplitude vibrations can be examined:

1. Pre-event loops: Assuming that the vibration amplitude is so low that yielding of the structure is not engaged before the event, the pivot points are the yielding points in both directions. The loops degrade to a straight line with a slope equal to the initial stiffness $k_{0}$; and

2. Postevent loops: If the event is damaging, yielding in either/ both directions might have occurred. Assuming again that the postevent vibration amplitude is small and no further damage is engaged (which implies that the largest excursion points ever reached in the damaging event have not been exceeded during the entire postevent low-amplitude vibration), both the pivot points are the largest excursion points in the positive and negative directions and they remain unchanged. Therefore the hysteresis goes through narrow loops (solid line in Fig. 2) that lay on top of the straight line connecting the two pivot points. A structure in such a vibration generally behaves as if a linear system with a degraded stiffness $\left(k_{a} \approx F_{m} / d_{m}\right)$ and a lightly increased damping ratio are compared to pre-event situations.

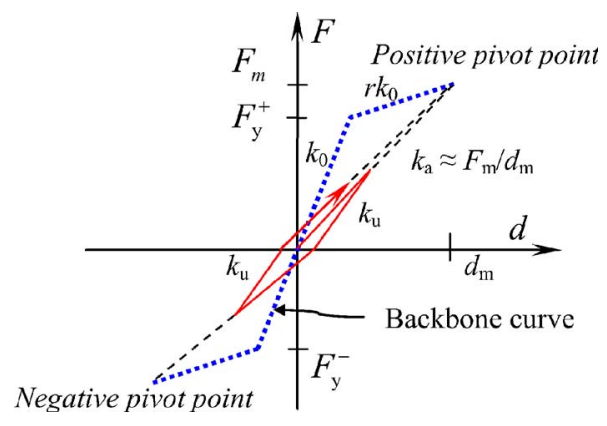

Fig. 2. Postevent low-amplitude hysteresis loops

This observation suggests that pre- and postevent lowamplitude vibrations can be treated as a LTI system, which significantly eases the identification of structural properties. Moreover, the postevent effective stiffness $k_{a}$ is meaningful if correctly identified based on vibration measurements, because it can be converted back to the largest excursion point during the damaging event, if a backbone curve is obtained by, e.g., a pushover analysis. The point of largest excursion contains crucial information to evaluate the residual strength and deformation capacity the damaged structure still possesses. This postulation is supported by experimental data to be discussed later.

A note shall be made here on the measurability of hysteresis loops. Hysteresis, by definition, is the relation of restoring force versus deformation of a structural component. Deformation can be readily measured by a displacement sensor or a strain gauge in structural tests. Direct measurement of restoring force, however, is not always available, except in static or pseudodynamic tests where load cells are used. In a dynamic test (i.e., shake table test) of a nonlinear multidegree-of-freedom (MDOF) system involving multiple structural components, the variation of relative stiffness among components during the test will cause force redistribution. As a result, the global inertial force is no longer proportional to the restoring force of an individual component. In such cases system identification methods can play an important part in constructing the hysteresis curve of a particular structural component.

It shall also be understood that, pertaining to structural condition is the hysteresis of critical structural components, not the global hysteresis of the entire structure. It has been documented in several lab and field tests that a structural assembly of components can vary its global stiffness in a considerable wide range without significant damage being observed. One explanation for this phenomenon is that the changes of the hysteresis of some uncritical components (such as loosening of an anchorage, or cracking or softening in some preselected regions, e.g., end zones of slab or gravity beams, which have been engineered to accommodate such plastic behaviors), are not impairing the overall structural performance and thus are not considered as damage to an assembly. The change of characteristics of uncritical components, however, is reflected in the global hysteresis of the assembly. For example, a gap developed due to an insignificant loosening in the anchor bolts could result in an apparent change in the hysteresis of a wall assembly, but the wall panel may still in a sound condition. Ideally for structural condition assessment, one should monitor the hysteresis changes of those components pertaining to structural performance, not the global hysteresis of an assembly. To do so, it is important to obtain from dynamic data the hysteresis for a selected component. 


\section{System Identification Methods}

In this section two system identification methods used in this study are outlined. They are model-based parametric methods that utilize global optimization to identify the model parameters that reconcile the predicted and measured vibration characteristics [i.e., frequencies and mode shapes (Method 1) or response time histories (Method 2)]. The system model is assumed LTI, and the identification method is relatively easy to implement by optimization routines.

\section{Model Parameterization: Sectional Stiffness Reduction Coefficients}

Actual sectional stiffness is represented by a set of correction coefficients, $\beta_{i}$ s, being a fraction of the sectional stiffness calculated from drawings. To be specific

$$
\beta_{i}=\frac{(k)_{A}^{i}}{(k)_{D}^{i}}
$$

where $(k)_{A}^{i}=$ actual (subscript $A$ ) sectional stiffness of element $i$; and $(k)_{D}^{i}=$ sectional stiffness of the same element calculated from drawings (subscript $D$ ). Sectional stiffness $k$ can be either $E I$ for bending stiffness or $E A$ for axial stiffness of an element, depending on the applications.

Rayleigh type damping was assumed in this study for all the models. A Rayleigh damping matrix $\mathbf{C}$ is a linear combination of the mass and stiffness matrices $\mathbf{M}$ and $\mathbf{K}$ by

$$
\mathbf{C}=a \mathbf{M}+b \mathbf{K}
$$

Two correction coefficients, $\alpha_{i}(i=1,2)$, quantifying the damping characteristics of the specimens are defined as

$$
\alpha_{1}=\frac{(a)_{A}}{(a)_{D}} \quad \text { and } \quad \alpha_{2}=\frac{(b)_{A}}{(b)_{D}}
$$

where $(a)_{A}=$ actual (subscript $A$ ) Rayleigh coefficient $a$; and $(a)_{D}=$ Rayleigh coefficient $a$ assumed for design purpose (subscript $D$ ); while $(b)_{A}=$ actual (subscript $A$ ) Rayleigh coefficient $b$ and $(b)_{D}=$ that for design purpose (subscript $\left.D\right) .(a)_{D}$ and $(b)_{D}$, following the general design practice, are assigned so that the finite-element model derived from design drawings has 5\% damping for both the first and the second modes.

With a finite-element model of the specimen parameterized by $\beta_{i} \mathrm{~s}$ and $\alpha_{i} \mathrm{~s}$, collectively denoted as

$$
\theta=\left\{\beta_{1}, \ldots, \beta_{\mathrm{n}}, \alpha_{1}, \alpha_{2}\right\}^{T}
$$

the analytical natural frequencies, mode shapes, and modal damping ratios are all readily obtained by eigenanalysis. These are used in the system identification Method 1 for the first experiment. For the second experiment, an alternative method, Method 2, is used for parameter identification. Method 2 uses a multiple-inputmultiple-output (MIMO) state-space formulation to predict the structural response time-histories and compare them to the measured signals, so as to avoid possible errors when extracting the modal characteristics (frequencies, mode shapes, and damping) from vibration data.

\section{MIMO State-Space Model Formulation}

If the structure has $n$ DOFs, they can be classified into two catalogs: (1) $n_{\mathrm{I}}$ DOFs associated with prescribed inputs (acceleration, velocity, displacement, or force time histories) and therefore sub- jected to external forces; and (2) $n_{f}$ other DOFs that are not associated with inputs nor subjected to external forces, "free" in this sense. The equations of motion are then partitioned as following by grouping the DOFs of these two catalogs denoted by subscript $I$ or $f$, respectively

$$
\begin{gathered}
{\left[\begin{array}{l|l}
M_{I I} & M_{I f} \\
\hline M_{f I} & M_{f f}
\end{array}\right]\left\{\frac{\ddot{X}_{I}}{\ddot{X}_{f}}\right\}+\left[\begin{array}{l|l}
C_{I I} & C_{I f} \\
\hline C_{f I} & C_{f f}
\end{array}\right]\left\{\frac{\dot{X}_{I}}{\dot{X}_{f}}\right\}+\left[\begin{array}{c|c}
K_{I I} & K_{I f} \\
\hline K_{f I} & K_{f f}
\end{array}\right]\left\{\frac{X_{I}}{X_{f}}\right\}} \\
=\left\{\begin{array}{c}
F \\
0
\end{array}\right\}
\end{gathered}
$$

Now we only address the case where inputs are specified by desired acceleration time histories. In this case, vector $F$ on the right hand site of Eq. (5), representing external forces (in this experiment, these forces are from the shake tables, transferred to the bases of the bents), is usually not directly available. Instead, the acceleration time-histories at the input DOFs are known, therefore, we rewrite the motion equations in

$$
\begin{aligned}
& {\left[\begin{array}{c|c}
I & 0 \\
\hline M_{f I} & M_{f f}
\end{array}\right]\left\{\frac{\ddot{X}_{I}}{\hdashline \ddot{X}_{f}}\right\}+\left[\begin{array}{c|c}
0 & 0 \\
\hline C_{f I} & C_{f f}
\end{array}\right]\left\{\frac{\dot{X}_{I}}{\dot{X}_{f}}\right\}+\left[\begin{array}{c|c}
0 & 0 \\
\hline K_{f I} & K_{f f}
\end{array}\right]\left\{\frac{X_{I}}{X_{f}}\right\}} \\
& =\left\{\frac{\ddot{X}_{I}}{0}\right\}
\end{aligned}
$$

Letting

$$
\bar{M}=\left[\begin{array}{c|c}
I & 0 \\
\hline M_{f I} & M_{f f}
\end{array}\right], \quad \bar{C}=\left[\begin{array}{c|c}
0 & 0 \\
\hline C_{f I} & C_{f f}
\end{array}\right], \quad \text { and } \quad \bar{K}=\left[\begin{array}{c|c}
0 & 0 \\
\hline K_{f I} & K_{f f}
\end{array}\right]
$$

and

$$
\bar{X}=\left\{\frac{X_{I}}{X_{f}}\right\} \quad \text { and } \quad \bar{U}=\left\{\frac{\ddot{X}_{I}}{0}\right\}
$$

a state-space representation of the structural dynamic system can be obtained by the standard procedure

$$
\left\{\begin{array}{c}
\dot{\bar{X}} \\
\ddot{\bar{X}}
\end{array}\right\}=\left[\begin{array}{cc}
0 & I \\
-\bar{M}^{-1} \bar{K} & -\bar{M}^{-1} \bar{C}
\end{array}\right]\left\{\begin{array}{c}
\bar{X} \\
\dot{\bar{X}}
\end{array}\right\}+\left\{\begin{array}{c}
0 \\
\bar{M}^{-1} \bar{U}
\end{array}\right\}
$$

Assuming that the experimental measurements are the acceleration responses at some of the DOFs, the observation matrix is $O$ such that $Z=O \cdot \ddot{\bar{X}}$, the measurement $Z$ is then

$$
Z=O\left[-\bar{M}^{-1} \bar{K}-\bar{M}^{-1} \bar{C}\right]\left\{\begin{array}{c}
\bar{X} \\
\dot{\bar{X}}
\end{array}\right\}+O \bar{M}^{-1} \bar{U}
$$

With this MIMO state-space model [Eqs. (7) and (8)], responses can be efficiently simulated. When the structure is parameterized, $M_{f I}, M_{f f}, C_{f I}, C_{f f}, K_{f I}$, and $K_{f f}$ are all argumented by the parameter $\theta$. Therefore, the MIMO state-space model is a good device to simulate the responses when the structure status is represented by a finite number of parameters, facilitating the system identification procedure. 


\section{Parameter Identification: Method 1}

A weighted-nonlinear-least-square procedure was used to identify $\alpha_{i}$ s and $\beta_{i}$ s (i.e., $\theta$ ) for the specimen in the first experiment, based on the modal characteristics. The following object function is employed

$$
\begin{aligned}
\operatorname{Obj}(\theta)= & \left(1,000 \cdot \Delta f_{1}\right)^{2}+\left(500 \cdot \Delta f_{2}\right)^{2}+\left(10 \cdot \mathrm{MAC}_{1}\right)^{2} \\
& +\left(\mathrm{MAC}_{2}\right)^{2}+\left(10 \cdot \Delta \zeta_{1}\right)^{2}+\left(\Delta \zeta_{2}\right)^{2}
\end{aligned}
$$

where

$$
\begin{gathered}
\Delta f_{i}=\frac{f_{i}^{M}-f_{i}(\theta)}{f_{i}^{M}}, \quad(i=1,2) ; \\
\operatorname{MAC}_{i}=\frac{\left[\left(\phi_{i}^{M}\right)^{T} \cdot \phi_{i}^{A}\right]^{2}}{\left[\left(\phi_{i}^{M}\right)^{T} \cdot\left(\phi_{i}^{M}\right)\right]\left[\left(\phi_{i}^{A}\right)^{T} \cdot \phi_{i}^{A}\right]}, \quad\left(i=1,2 ; \text { and } \phi_{i}^{A}=\phi_{i}(\theta)\right)
\end{gathered}
$$

and

$$
\Delta \zeta_{i}=\frac{\zeta_{i}^{M}-\zeta_{i}(\theta)}{\zeta_{i}^{M}}, \quad(i=1,2)
$$

$f_{i}^{M}, \phi_{i}^{M}$, and $\zeta_{i}^{M}=$ natural frequency, mode shape, and damping ratio of the $i$ th mode extracted from vibration measurements, respectively; $f_{i}(\theta), \phi_{i}(\theta)$, and $\zeta_{i}(\theta)=$ analytical frequency, mode shape, and damping ratio of the $i$ th mode associated with a correction coefficient set $\theta$. MAC values $=$ indicators of the similarity between two shapes and different weights, such as 1,000, 500, 10, and 1 , are adopted to emphasize the relative significance among the vibration characteristics and the various confidence levels when they are obtained from the measured data. Parameters in $\theta$, $\left(\beta_{1}, \beta_{2}, \alpha_{1}, \alpha_{2}\right)$, are each confined to a lower bound 0.001 and an upper bound 4 , based on a priori knowledge of the structural system. To solve the weighted-nonlinear-least-square problems, a minimization with linear constrains by the quasi-Newton method (Polak 1997) is employed.

\section{Parameter Identification: Method 2}

A similar weighted-nonlinear-least-square procedure was used to identify $\alpha_{i}$ s and $\beta_{i}$ s (i.e., $\theta$ ) for the specimen in the second experiment. The object function in Method 2 is

$$
\operatorname{Obj}(\theta)=(\Delta \mathbf{Z})^{\mathbf{T}} \mathbf{W}(\Delta \mathbf{Z})
$$

where $\Delta \mathbf{Z}=\mathbf{Z}^{M}-\mathbf{Z}(\theta)$. $\mathbf{Z}^{M}=$ matrix containing the measured timehistories of the acceleration responses at the sensor locations, arranged in such a way that its columns are associated with different channels and rows are associated with increasing time. $\mathbf{Z}(\theta)=$ matrix of simulated acceleration responses using the MIMO state-space model parameterized by $\theta$, arranged in the same manner as $\mathbf{Z}^{M}$. $\mathbf{W}=$ inverse matrix of the covariance of the noises in different channels, so that the channels with lower noise level are trusted more than those with higher noise level. The identification of sectional stiffness reduction is now optimizing $\theta$ to get a minimum of the object function. Parameters in $\theta,\left(\beta_{1}, \beta_{2}, \beta_{3}, \alpha_{1}, \alpha_{2}\right)$ are each confined to a lower bound 0.001 and an upper bound 4 , based on a priori knowledge of the structural system. To reduce the risk of converging to a local minimum, 500-1,000 random searches are performed to get a globally plausible initial set of $\theta^{\circ}$. In each of the random search, $\beta_{i}^{0} \mathrm{~s}$ and $\alpha_{i}^{0} \mathrm{~s}$ are randomly picked from uniform distributions between their lower and upper bounds. The smallest $\operatorname{Obj}(\cdot)$ is registered and the associated set of $\left(\beta_{1}^{0}, \beta_{2}^{0}, \beta_{3}^{0}, \alpha_{1}^{0}, \alpha_{2}^{0}\right)$ is adopted as the initial values for quasi-Newton optimization. The quasi-Newton optimization

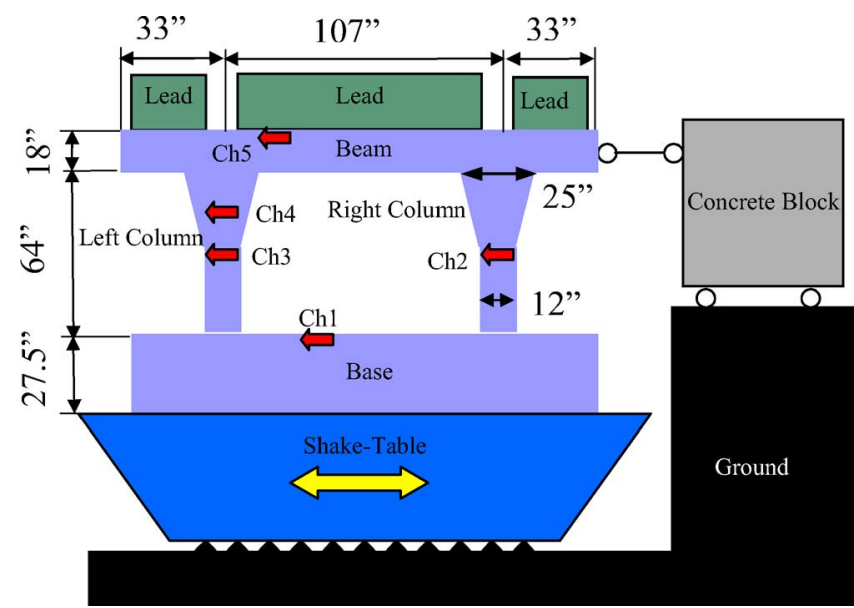

Fig. 3. Schematic plot of Experiment 1

further refines the parameters, and obtains the parameters that best reconcile the simulated and measured responses.

\section{Experimental Setup, Procedure, and Observation}

The experimental setups, test procedures and damage observed in the two experiments conducted at UNR are described in this section. The data processing and parameter identification will be presented in the next section.

\section{Experiment 1: Two-Column Bent Specimen}

Experiment 1 was performed on a flared two-column reinforced concrete (RC) bent specimen (Fig. 3), with column height $1.63 \mathrm{~m}$ (64 in.) and bent beam length $4.39 \mathrm{~m}$ (177 in.). As sketched in Fig. 3, to simulate the mass of the superstructure, two compensative masses were added: one 60 kips composed of lead placed on the bent beam and another 40 kips composed of the concrete block set aside but linked to the beam by a steel rod. Acceleration signals from the five sensors (Fig. 3) were recorded.

The ground motion record at Sylmar station in the 1994 Northridge Earthquake was used as the driving signal of the shake table to simulation strong motion. Its amplitude was scaled with different scaling factors, namely $0.15,0.25,0.50,0.75,1.00,1.25$, $1.50,1.75,2.00,2.50$, and 2.75 , respectively, in sequential tests to introduce different levels of damage. Before and after each of the strong motions, an ambient input measured at a real bridge, amplified by 50 times just to overcome the friction of the shake table, drove the shake table to perturb the bent specimen in the corresponding damage level. Four small amplitude free vibrations were also performed to provide another mechanism for identifying the system characteristics at different damage levels.

During the test, after each level of the strong motions, cracks were marked and photos were taken to document the damage. Fig. 4 shows the damage observed in the flared portion of the columns. As the damage accumulated, more and more cracks were observed. However, by looking only at the crack distribution, it is hard to quantify the damage. It is also difficult to correlate the crack patterns with the strength or deformation reservation the bent has before the onset of a catastrophic collapse. 

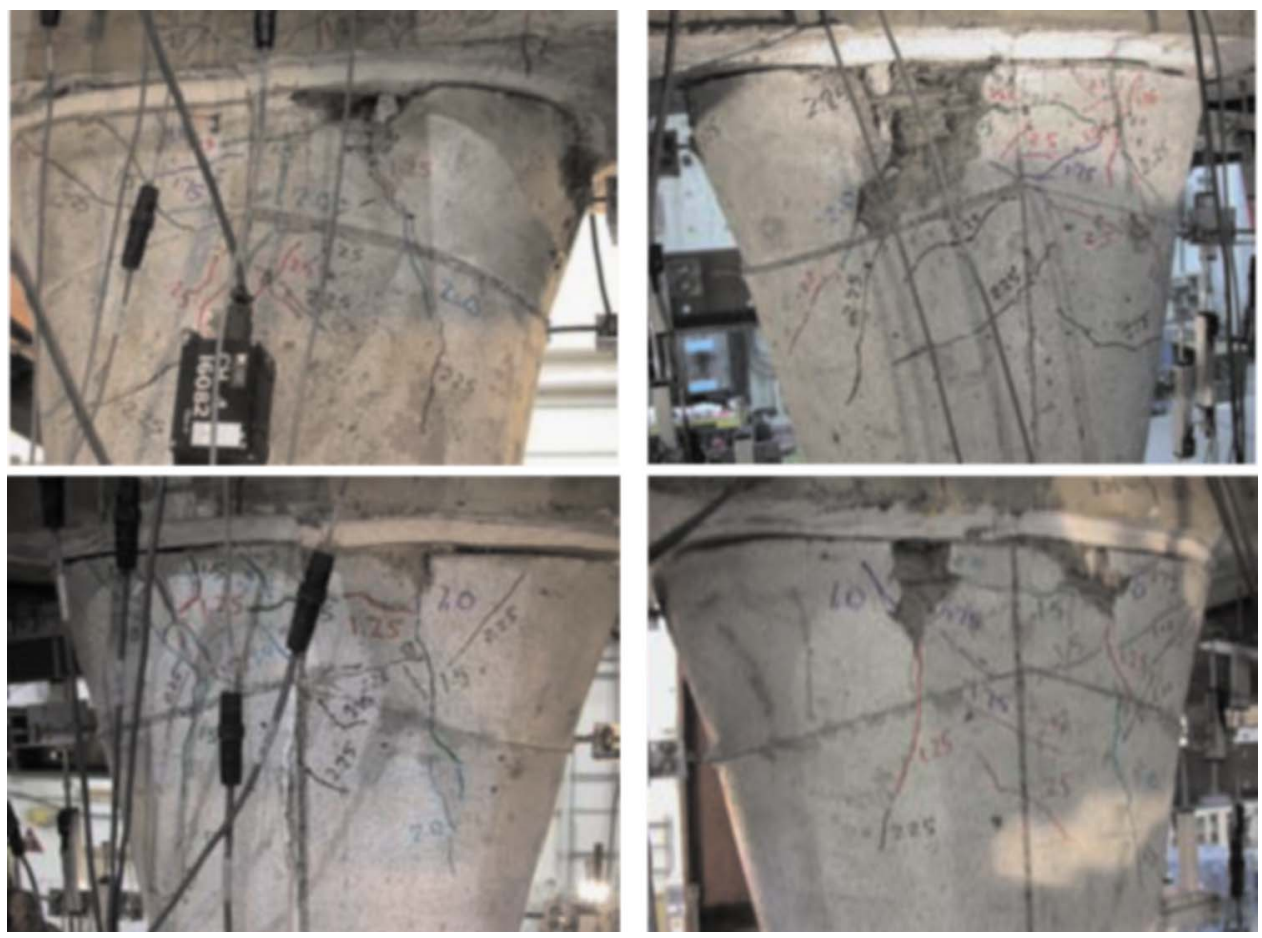

Fig. 4. Damage at flared portion of columns

\section{Experiment 2: Two-Span Three-Bent Bridge Specimen}

Experiment 2 was performed on a two-span three-bent RC bridge specimen. As shown in Fig. 5, each of the three bents is supported on an individual shake table. The bents are linked by the bridge deck, with total length $18.29 \mathrm{~m}$ (720 in.), which consists of three post-tensioned beams. Each of the bents has two columns, having the same design cross sections with diameter $0.3 \mathrm{~m}$ (12 in.). The bents are of different heights, $1.83 \mathrm{~m}$ (72 in.), $2.44 \mathrm{~m}$ (96 in.) and $1.52 \mathrm{~m}$ (60 in.) for Bents 1, 2, and 3, respectively, so that they process significantly different transverse stiffness. To resemble the inertia of other parts of the superstructure not built into this specimen, compensative masses were added. The shake tables were driven by input acceleration signals in the transverse direction of the bridge. Eleven accelerometers were installed on the specimen to obtain the acceleration inputs and responses of the bridge, as illustrated in Fig. 5. Note that the deck is divided into two simply supported spans in the vertical bending direction. However, in the transverse direction, the existence of parallel post-tension tendons warrants a continuous bending moment transmission.

During the tests, various earthquake ground motions tailored to various soil-foundation scenarios were used as the driving signals of the shake tables to simulate strong motions. By their demands to the bridge structure, ground motions were classified into different levels, such as low, moderate, high, severe, and extreme. After the most strong ground motion, a smaller motion was input to mimic an after-shot earthquake. In Table 1, the sequence of the tests (denoted in Table 1 by 'T-\#') and their input peak ground accelerations (PGA) are listed. Different levels of damage were introduced to the bridge specimen by these strong motions. In between of the strong motions, low-amplitude white noise (PGA is approximately $0.05 g$ ) drove the shake tables to

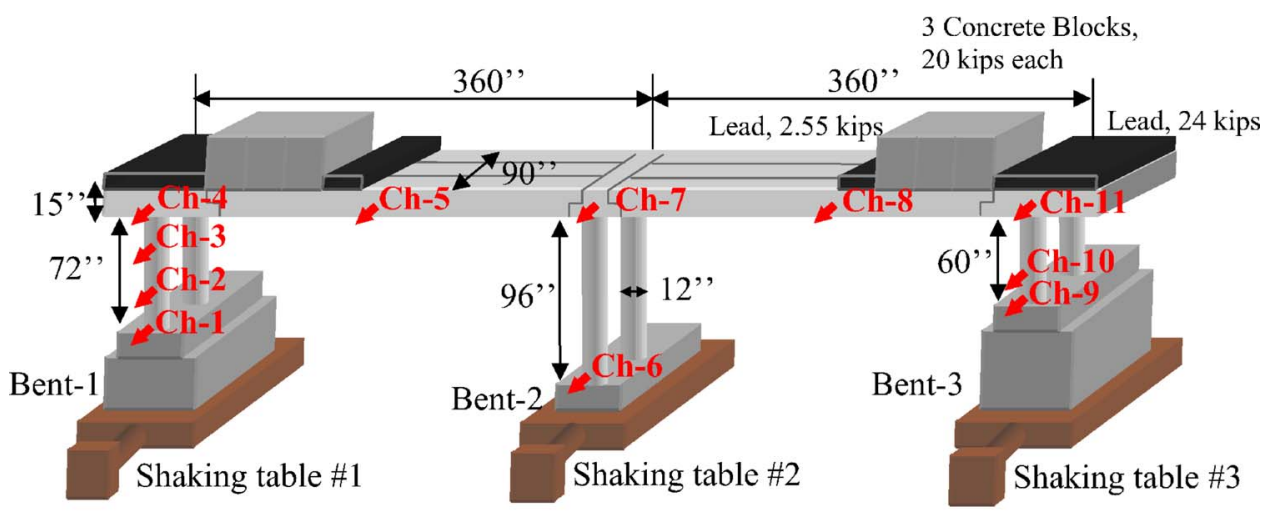

Accelerometer

Fig. 5. Design and sensor layout of bridge specimen in Experiment 2 


\begin{tabular}{|c|c|c|c|}
\hline Tests & Ground motion description & $\begin{array}{c}\text { PGA } \\
(g)\end{array}$ & $\begin{array}{c}\text { Damage } \\
\text { description }\end{array}$ \\
\hline S-1 & Snap (arouse free vibration) & - & - \\
\hline $\mathrm{WN}-\mathrm{X}-1$ & White noise in transverse & - & - \\
\hline WN-Y-1 & White noise in longitudinal & - & - \\
\hline $\mathrm{T}-12$ & Low earthquake in transverse & 0.0851 & - \\
\hline $\mathrm{T}-13$ & Low earthquake in transverse & 0.1729 & Bent 1 yields \\
\hline $\mathrm{T}-14$ & Moderate earthquake in transverse & 0.3193 & Bent 3 yields \\
\hline $\mathrm{S}-2$ & Snap (arouse free vibration) & - & - \\
\hline $\mathrm{WN}-\mathrm{X}-2$ & White noise in transverse & - & - \\
\hline WN-Y-2 & White noise in longitudinal & - & - \\
\hline $\mathrm{T}-15$ & High earthquake in transverse & 0.6272 & Bent 2 yields \\
\hline $\mathrm{T}-16$ & Severe earthquake in transverse & n.a. ${ }^{a}$ & - \\
\hline $\mathrm{T}-17$ & Extreme earthquake in transverse & 1.135 & - \\
\hline $\mathrm{S}-3$ & Snap (arouse free vibration) & - & - \\
\hline $\mathrm{WN}-\mathrm{X}-3$ & White noise in transverse & - & - \\
\hline WN-Y-3 & White noise in longitudinal & - & - \\
\hline $\mathrm{T}-18$ & Extreme earthquake in transverse & 1.3975 & - \\
\hline $\mathrm{T}-19$ & Extreme earthquake in transverse & 1.7033 & Bent 3 steel buckles \\
\hline S-4 & Snap (arouse free vibration) & - & - \\
\hline $\mathrm{WN}-\mathrm{X}-4$ & White noise in transverse & - & - \\
\hline WN-Y-4 & White noise in longitudinal & - & - \\
\hline $\mathrm{T}-20$ & After shot in transverse & 1.2861 & - \\
\hline S-5 & Snap (arouse free vibration) & - & - \\
\hline $\mathrm{WN}-\mathrm{X}-5$ & White noise in transverse & - & - \\
\hline WN-Y-5 & White noise in longitudinal & - & - \\
\hline
\end{tabular}

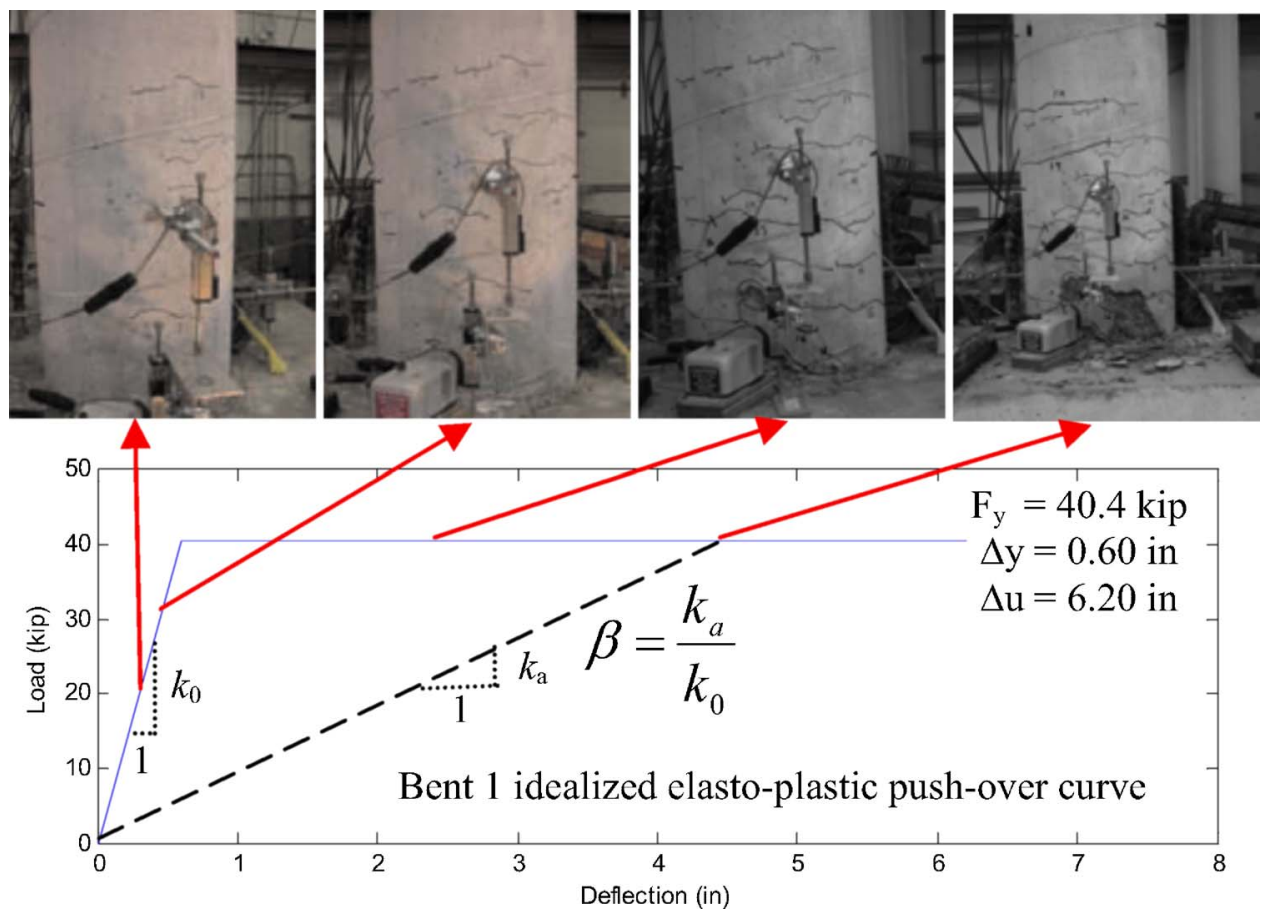

Fig. 6. Damage observed at column of Bent 1 


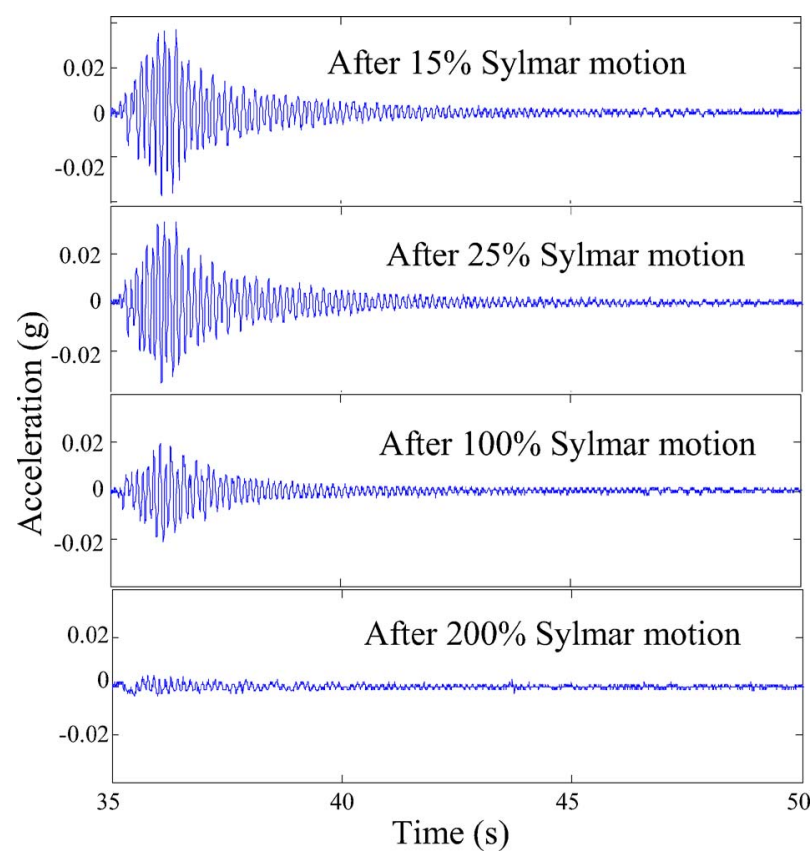

Fig. 7. Measured acceleration responses at Ch-5 to ambient excitations

perturb the specimen in the corresponding damage level (denoted by 'WN-X-\#' or 'WN-Y-\#' in Table 1, where $\mathrm{X}$ denotes the excitations along the transverse direction of the bridge, and $\mathrm{Y}$ denotes those in the longitudinal direction). Free vibrations were also aroused by inputting a snap wave (denoted by ' $\mathrm{S}$-\#' in the table).

Fig. 6 shows the crack propagation at the lower portion of a column of Bent 1. As the damage accumulated, more and more cracks were observed, and finally concrete spallings were seen at the bottom of the column.

It is observed that cracks are not a clear indication of the formation of a plastic hinge. In this experiment, advantage was taken of the densely instrumented strain gauges on the steel rebars embedded before concrete casting to read the deformation of the steels, based on which yielding of the bents was found. As indicated in Table 1, the damage procedure observed can be outlined as: Bent 1 yields $\rightarrow$ Bent 3 yields $\rightarrow$ Bent 2 yields $\rightarrow$ Bent 3 steel buckles. This procedure is largely determined by the relative heights of the bents. The onset of Bent 1 yielding is due to the fact that the first mode of this bridge specimen (in its undamaged stage) has the largest displacement on Bent 1 . After the yielding of Bent 1, Bent 3 attracts the most seismic force and yields, and this also happens to Bent 2 after the yielding of Bents 1 and 3 . The final collapse (in the test, the specimen was protected to avoid actual collapse) is associated with the steel buckling at Bent 3 , which has the smallest ductility capacity among the three.

On real-life bridges, however, such dense strain gauge installation is hardly possible. On the contrary, the sectional stiffness reduction obtained by system identification based on vibration measurement requires only minor instrumentation efforts (compared with strain gauge installation) and can be implemented on an already existing structure. As will be illustrated later, the reduction (represented by correction coefficients of sectional stiffness) correlates satisfactorily with the damage procedure.

\section{Vibration Measurement and System Identification Results}

The accelerations in both experiments were digitized and recorded. The sampling frequency was set to $200 \mathrm{~Hz}$. The processing and the system identification results will be presented.

\section{Experiment 1}

The ambient perturbations at the base of the bent were scaled from a record on an instrumented three-span RC box-girder highway bridge at Irvine, Calif. It has dominating energy in the range between 5 and $10 \mathrm{~Hz}$. The specimen varied its responses to this ambient input at different damage stages, as shown by a portion of the response time-histories depicted in Fig. 7. The specimen behaved linearly before damage occurred (after 15 and $25 \%$ Sylmar motions), while the amplitudes and the frequencies were reduced as damage occurred and accumulated.

Shown in columns marked as "measured" in Table 2, natural frequencies, mode shapes, and damping ratios of the first two modes are obtained by peakpicking the power spectrum curves and the conventional half-power method. Note that the identification of damping characteristics is not reliable, because viscous damping cannot fully represent the nature of hysteresis behaviors.

Table 2. Comparison of Modal Characteristics

\begin{tabular}{|c|c|c|c|c|c|c|c|c|c|c|c|c|}
\hline & \multicolumn{2}{|c|}{ After $15 \%$ Sylmar } & \multicolumn{2}{|c|}{ After $25 \%$ Sylmar } & \multicolumn{2}{|c|}{ After $50 \%$ Sylmar } & \multicolumn{2}{|c|}{ After $100 \%$ Sylmar } & \multicolumn{2}{|c|}{ After $200 \%$ Sylmar } & \multicolumn{2}{|c|}{ After $275 \%$ Sylmar } \\
\hline & Measured & Analytical & Measured & Analytical & Measured & Analytical & Measured & Analytical & Measured & Analytical & Measured & Analytical \\
\hline$f_{1}(\mathrm{~Hz})$ & 4.25 & 4.11 & 4.15 & 4.0329 & 3.88 & 3.82 & 3.47 & 3.47 & 1.76 & 1.76 & 1.46 & 1.47 \\
\hline$f_{2}(\mathrm{~Hz})$ & 7.59 & 8.46 & 7.54 & 8.2981 & 7.47 & 7.86 & 7.57 & 7.57 & 7.50 & 7.52 & 7.42 & 7.42 \\
\hline \multirow[t]{4}{*}{$\phi_{1}$} & -0.5000 & -0.0163 & -0.5214 & -0.0166 & -0.5523 & -0.0170 & -0.5649 & -0.0220 & -0.4063 & -0.0288 & -0.4060 & 0.0292 \\
\hline & -0.3587 & -0.0167 & -0.3633 & -0.0170 & -0.3372 & -0.0175 & -0.3443 & -0.0228 & -0.3715 & -0.0301 & -0.3882 & 0.0304 \\
\hline & -0.4919 & -0.0269 & -0.4794 & -0.0274 & -0.4621 & -0.0282 & -0.4642 & -0.0366 & -0.5526 & -0.0482 & -0.5320 & 0.0488 \\
\hline & -0.6152 & -0.0331 & -0.6024 & -0.0337 & -0.6010 & -0.0345 & -0.5885 & -0.0447 & -0.6253 & -0.0584 & -0.6286 & 0.0591 \\
\hline \multirow[t]{4}{*}{$\phi_{2}$} & -0.5722 & 0.0339 & -0.5738 & 0.0337 & -0.5625 & 0.0336 & -0.5616 & -0.0307 & -0.3892 & -0.0248 & -0.3860 & 0.0245 \\
\hline & -0.3371 & 0.0356 & -0.3389 & 0.0355 & -0.3402 & 0.0353 & -0.3380 & -0.0324 & -0.3779 & -0.0265 & -0.3767 & 0.0261 \\
\hline & -0.4521 & 0.0573 & -0.4510 & 0.0571 & -0.4537 & 0.0568 & -0.4493 & -0.0521 & -0.5042 & -0.0423 & -0.4961 & 0.0417 \\
\hline & -0.5952 & 0.0699 & -0.5924 & 0.0696 & -0.6016 & 0.0692 & -0.5918 & -0.0631 & -0.6709 & -0.0507 & -0.6804 & 0.0498 \\
\hline$\zeta_{1}(\%)$ & 1.77 & 1.77 & 3.43 & 3.40 & 2.10 & 2.10 & 9.30 & 9.07 & 3.29 & 3.29 & 2.05 & 2.05 \\
\hline$\zeta_{2}(\%)$ & 2.12 & 2.15 & 1.15 & 1.68 & 1.34 & 1.36 & 1.92 & 4.15 & 10.43 & 10.66 & 15.30 & 10.24 \\
\hline
\end{tabular}




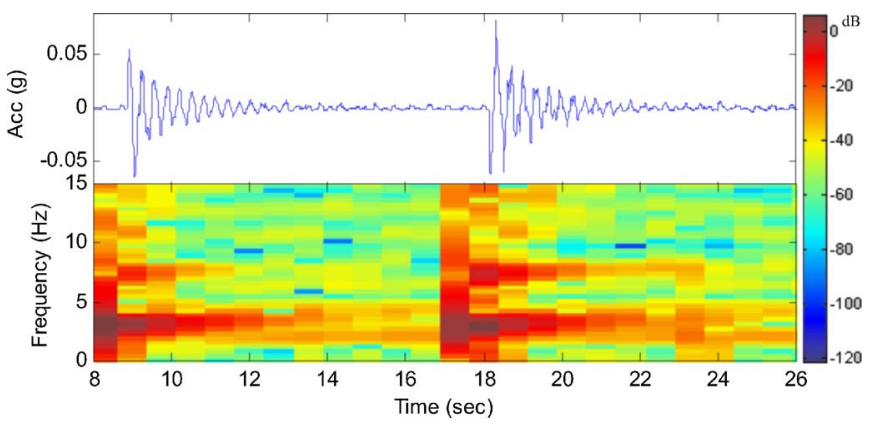

Fig. 8. Free vibration II (after 100\% Sylmar) and its time-frequency plot

Fig. 8 shows the time-frequency plots by short-time Fourier transform of the free vibration response at Ch-5 after 100\% Sylmar motion. It illustrates, to some extent, the intrinsic difficulty of time-frequency analysis which intend to trace the structure changes at every instance, i.e., either the frequency resolution or the time resolution have to be compromised (Chen and Feng 2003).

Using Method 1 outlined previously, the correction coefficients for the column bending stiffness and the Rayleigh damping coefficients at each damage stage are obtained as in Table 3. The sectional stiffness drops dramatically as damage accumulated, as indicated by $\beta_{1}$ decreasing from 0.91 all the way to 0.07 . Table 2 compares the experimental modal characteristics to their analytical counterparts of the identified models.

\section{Experiment 2}

To lessen the effect of noise, signals were band-pass filtered in frequency range from 1 to $15 \mathrm{~Hz}$. Fig. 9 shows the filtered acceleration at Ch-4 during low-amplitude transverse white noise tests.

Following the Method 2 outlined above, the correction coefficients are identified at different damage stages using the acceleration measurements obtained in the low-amplitude white noise tests. The results are listed in Table 4. Fig. 10 shows that the identified sectional stiffness coefficients clearly indicate the same damage procedure as observed in the experiment. Bent 1 yields $\rightarrow$ Bent 3 yields $\rightarrow$ Bent 2 yields $\rightarrow$ Bent 3 steel buckles. Between WN-X-1 and WN-X-2, $\beta_{1}$ and $\beta_{3}$ drop from 0.78 to 0.52 and from 0.84 to 0.61 respectively, while $\beta_{2}$ remains at the same level, indicating in a quantitative manner the yielding of Bent 1 and Bent 3 between these two tests. Then between WN-X-2 and $\mathrm{WN}-\mathrm{X}-3$, the decrements in all $\beta_{1}, \beta_{2}$, and $\beta_{3}$ signal that not only Bent 2 yielded, but also that the damage in Bents 1 and 3 further developed. In $\mathrm{WN}-\mathrm{X}-4, \beta_{3}$ touches down to a very low value, 0.11 , associating with the severe damage in Bent 3 (steel buckling) and the results of $\mathrm{WN}-\mathrm{X}-5$ are comparable to those in $\mathrm{WN}$ -

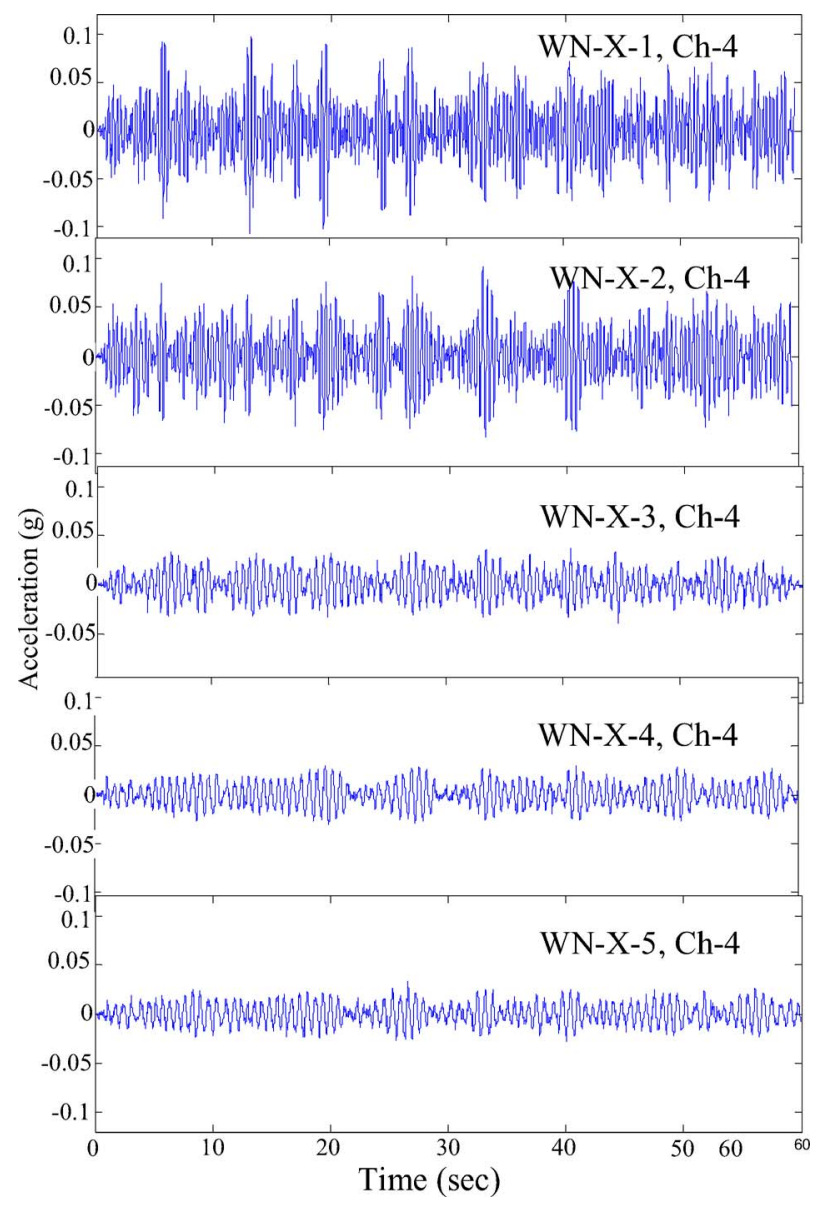

Fig. 9. Acceleration responses at $\mathrm{Ch}-4$ to white noise disturbances at various damage stages

$\mathrm{X}-4$, which is consistent with the observation that the after-shot earthquake actually had not further damaged the bridge specimen significantly.

To further verify the identified results, a MIMO model with proper corrections made according to the identified $\left(\beta_{1}, \beta_{2}, \beta_{3}\right.$, $\left.\alpha_{1}, \alpha_{2}\right)$ was used to simulate the specimen responses to WN-X-2 and WN-X-5 ground motions in time domain. They capture the primary characteristics of the bridge specimen response, as evident in the time-history simulation shown in Fig. 11.

Based on previous discussion on hysteresis behavior in postevent low-amplitude vibration, $\beta_{i}$ (in both experiments) is a quantitative indicator of the structural condition of the bent, which can be plausibly postulated as the secant sectional stiffness at the associated damage stage normalized by the initial sectional stiffness (see Figs. 2 and 6). If a pushover analysis is performed and a capacity curve of the specimen is obtained, $\beta_{i}$ can be further

Table 3. Identified Correction Coefficients for Experiment 1

\begin{tabular}{lccccc}
\hline & $\begin{array}{c}\text { After 15\% } \\
\text { Sylmar }\end{array}$ & $\begin{array}{c}\text { After 25\% } \\
\text { Sylmar }\end{array}$ & $\begin{array}{c}\text { After 50\% } \\
\text { Sylmar }\end{array}$ & $\begin{array}{c}\text { After 100\% } \\
\text { Sylmar }\end{array}$ & $\begin{array}{c}\text { After 200\% } \\
\text { Sylmar }\end{array}$ \\
\hline$\beta_{1}$ & 0.9120 & 0.8651 & 0.7600 & 0.5096 & 0.1035 \\
$\alpha_{1}$ & 0.2632 & 0.9249 & 0.4874 & 2.1275 & 0.1004 \\
$\alpha_{2}$ & 0.5561 & 0.0100 & 0.1569 & 0.0001 & 3.8730 \\
\hline
\end{tabular}


Table 4. Identified Correction Coefficients for Experiment 2

\begin{tabular}{lccccc}
\hline Tests & $\beta_{1}$ & $\beta_{2}$ & $\beta_{3}$ & $\alpha_{1}$ & $\alpha_{2}$ \\
\hline WN-X-1 & 0.78 & 0.79 & 0.85 & 1.02 & 2.05 \\
WN-X-2 & 0.53 & 0.80 & 0.61 & 0.33 & 6.24 \\
WN-X-3 & 0.19 & 0.31 & 0.18 & 0.82 & 3.52 \\
WN-X-4 & 0.20 & 0.16 & 0.11 & 2.12 & 1.28 \\
WN-X-5 & 0.17 & 0.13 & 0.13 & 0.85 & 4.21 \\
\hline
\end{tabular}

correlated to the deformation capacity reservation the specimen/ element has at a damage stage. This can be better seen later when experimental hysteresis is examined.

\section{Validation with Experimental Hysteresis Records}

Both the LTI assumption and the identified results find support in experimental observation. Plots in Fig. 12 are generated using data from the same tests in Experiment 2 described above. But these data were recorded and processed by another team, the researchers at UNR (Johnson et al. 2006), using a different set of sensors (that measured the force and the relative displacement on the top of the bent) than those deployed by the writers. The writers downloaded the UNR data from the NEES database and replotted them in a way to reveal the hysteresis during lowamplitude vibration, in order to validate the proposed LTI assumption and the identified stiffness reduction coefficient.

The recorded time-history of the force exerting on Bent 3 of the bridge specimen during T-13, T-14, T-15, and T-19 was first plotted. Then a threshold of \pm 20 kips was selected and the record of each test was divided into three segments. The first segment, e.g., Segment $(a)$ in T-13, is the "pre-event" segment, which starts when the test began and ends when the exerting force magnitude first exceeded the selected threshold. The second segment, e.g., Segment $(b)$ in T-13, is the "event" segment, which follows the pre-event segment and lasts until the force magnitude was retained smaller than the threshold in that test. Then the third segment, follows e.g., Segment (c) in T-13, the "postevent" segment. In both pre- and postevent segments, the bent experienced low-amplitude vibration, while in the event segment the vibration amplitude can be high enough to introduce damage to the bent. Twelve segments, Segments $(a)-(l)$, are thus defined from the four test records. In each of the segments, the record of exerting force is plotted versus the synchronized displacement record, resulting in the hysteresis curves $(a)-(l)$ in Fig. 12 for Segments $(a)-(l)$, respectively. In contrast to the hysteresis in the event segments, particularly Segment $(k)$, where the instantaneous stiffness went through abrupt changes, the hysteresis curves during

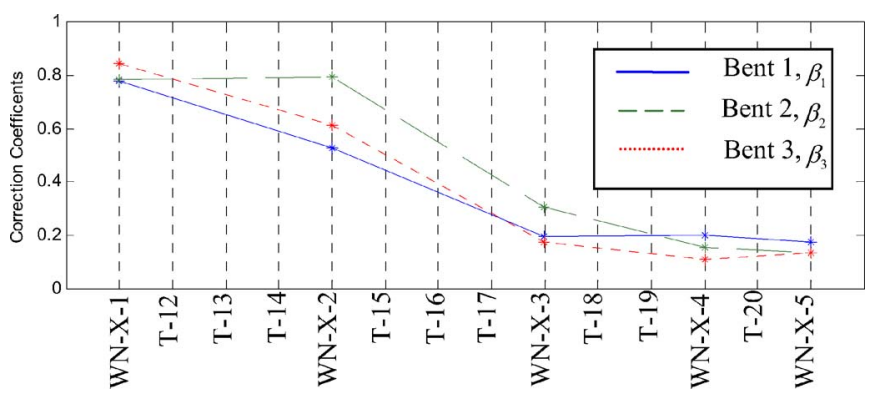

Fig. 10. Identified sectional stiffness coefficients
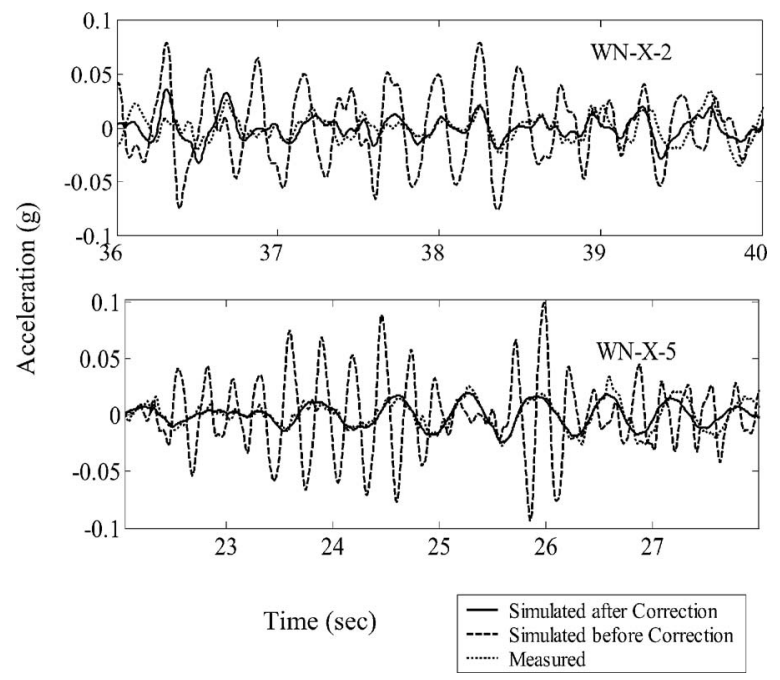

Fig. 11. Simulated and measured Ch-4 responses for $\mathrm{WN}-\mathrm{X}-2$ and WN-X-5 motions

low-amplitude vibration, in both pre- and postevent segments, exhibit very narrow loops and are apparently single lines in graphs $(a),(c),(d),(f),(g),(i),(j)$, and $(l)$ in Fig. 12. This suggests that the system behavior under low-amplitude vibration can be described with acceptable accuracy by a LTI model. A closer examination of the hysteresis curves in Fig. 12 reveals that, although the system appears to have some self-healing capacity and starts with similar initial stiffness in all tests in the pre-event, Segments $(a),(d),(g)$, and $(f)$, the slopes (equivalent stiffness) of hysteresis curves in the postevent segments, Segments $(c),(f)$, $(i)$, and $(l)$, do indicate the accumulated damage of the bent. The stiffness decreases between tests and the curves point to the largest excursion points the bent experienced in the events, as it is predicted by the pivot rule. Furthermore, if the relative ratio of slopes in Segments $(c),(f),(i)$, and $(l)$ are compared with the identified sectional stiffness reduction coefficient $\beta_{3}$ shown in Table 4, the identified results show good consistency with the experimental hysteresis.

\section{Conclusions}

This study conceptually justified methods based on LTI systems that identify structural component stiffness degradation using pre- and postevent low-amplitude vibration measurements, by examining the hysteresis loops a structural component experiences in low-amplitude vibrations. Two large-scale shake table experiments, one on a two-column RC bridge bent specimen, and the other on a two-span three-bent RC bridge specimen were performed. Progressive damages in specimens were introduced by subjecting them to earthquake ground motions with increasing amplitude. Low-amplitude vibration data in each of the damaged stages are analyzed by optimizing the parameters in a LTI model, and the postevent component stiffness correction coefficients were identified. The stiffness degradation identified is consistent with the experimental hysteresis, and could be quantitatively related to capacity residual of the components. The value of the proposed method lays essentially on its simplicity, and it is subjected to limitations. To name a few: compared to strong vibration, measurement accuracy might be worse in low-amplitude motions, and nonstructural effects (environmental temperature 

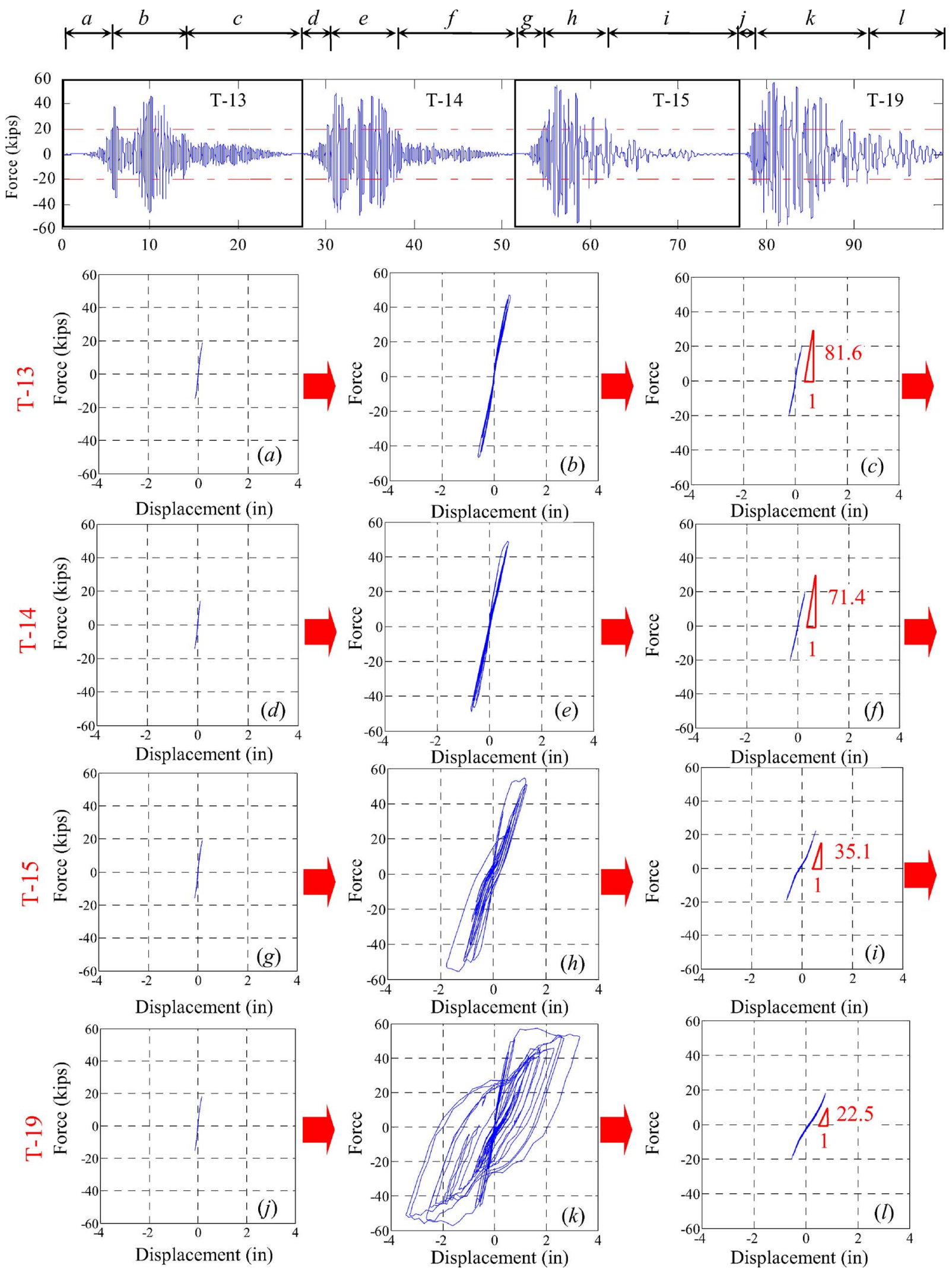

Fig. 12. Experimental hysteresis curves 
variation, for example) might be more substantial for lowamplitude vibration.

\section{Acknowledgments}

This study is sponsored by Caltrans under Contact No. 59A0311 managed by Mr. L.-H. Sheng. The shake table tests were conducted at University of Nevada, Reno by a research team at UNR. Deep appreciation is owed to Professor M. Saiidi, Professor D. Sendars, Dr. P. Laplace, and Mr. P. Lucas for their kind assistance during the experiments and for providing their design drawings, pushover analysis results, and some experimental data.

\section{References}

Bouc, R. (1967). "Forced vibration of mechanical systems with hysteresis." Proc., 4th Conf. on Non-Linear Oscillations, Prague, Czechoslovakia.

Chen, Y., and Feng, M. Q. (2003). "A technique to improve the empirical mode decomposition in the Hilbert-Huang transform." Earthquake Eng. Eng. Vibration, 2(1), 75-85.

Clough, R. W. (1966). "Effects of stiffness degradation on earthquake ductility requirement." Rep. No. 6614, Structural and Material Research Dept., Univ. of California, Berkeley, Calif.

Emori, K., and Schnobrich, W. C. (1981). "Inelastic behavior of concrete frame-wall structures." J. Struct. Div., 107(1), 145-164.

Johnson, N. S., Saiidi, M., and Sanders, D. H. (2006). "Large-scale ex- perimental and analytical seismic studies of a two-span reinforced concrete bridge system." Rep. No. CCEER-06-02, Center for Civil Engineering Earthquake Research, Dept. of Civil and Environmental Engineering/258, Univ. of Nevada Reno, Reno, Nev.

Park, Y. J., Reinhorn, A. M., and Kunnath, S. K. (1987). "IDARC: Inelastic damage analysis of reinforced concrete frame-shear-wall structures." Tech. Rep. No. NCEER-87-0008, State Univ. of New York at Buffalo, Buffalo, N.Y.

Polak, E. (1997). Optimization: Algorithms and consistent approximations, Springer, New York.

Saiidi, M. (1982). "Hysteresis models for reinforced concrete." J. Struct. Div., 108(5), 1077-1087.

Sain, P. M., Sain, M. K., and Spencer, B. F. (1997). "Models of hysteresis and application to structural control." Proc., American Control Conf., $16-20$.

Sivaselvan, M. V., and Reinhorn, A. M. (2000). "Hysteretic models for deteriorating inelastic structures." J. Eng. Mech., 126(6), 633-640.

Smyth, A. W., Masri, S. F., Kosmatopoulos, E. B., Chassiakos, A. G., and Caughey, T. K. (2002). "Development of adaptive modeling techniques for nonlinear hysteretic systems." Int. J. Non-Linear Mech., 37(8), 1435-1451.

Takayanagi, T., and Schnobrich, W. C. (1979). "Nonlinear analysis of coupled wall systems." Earthquake Eng. Struct. Dyn., 7, 1-22.

Takeda, T., Sozen, M. A., and Nielsen, N. N. (1970). "Reinforced concrete response to simulated earthquakes." J. Struct. Div., 96(12), 2557-2573.

Wen, Y.-K. (1976). "Method for random vibration of hysteretic systems." J. Engrg. Mech. Div., 102(2), 249-263.

Yang, J. N., and Lin, S. (2005). "Identification of parametric variations of structures based on least squares estimation and adaptive tracking technique.” J. Eng. Mech., 131(3), 290-298. 